J.G. Bolton

Division of Radiophysics, CSIRO, Sydney, Australia

Surveys of the sky between declinations $+25^{\circ}$ and $-90^{\circ}$ at $2700 \mathrm{MHz}$ $(11 \mathrm{~cm})$ have been in progress for the past 10 years. Excluding some regions close to the galactic plane the whole sky south of $+25^{\circ}$ has been surveyed to a flux density limit of $0.6 \mathrm{Jy}$ at $2700 \mathrm{MHz}$ and within this area surveys to limits of $0.35,0.25$ or $0.1 \mathrm{Jy}$ have been made covering $3.5 \mathrm{sr}$. Flux densities have been measured at $5000 \mathrm{MHz}$ for all sources stronger than $0.35 \mathrm{Jy}$ at $2700 \mathrm{MHz}$. The source positions have an average accuracy of $10^{\prime \prime}$ arc in both coordinates and the positions have been examined for optical identifications on Palomar, ESO or SRC sky survey plates, which now cover $95 \%$ of the area. The first part of this paper concerns the relationships between the spectral indices $\alpha(2700$ to $5000 \mathrm{MHz})$ and the identifications of the 2300 sources with galactic latitudes greater than $10^{\circ}$. It is a statistically significant sample, since the sources stronger than $0.35 \mathrm{Jy}$ cover $3.5 \mathrm{sr}$. It is also a representative sample, since no selection was made on the basis of spectral index or identification. It cannot however be claimed as a complete sample, for two reasons. A substantial fraction of sources found in radio surveys at high frequencies are variable - variations of up to a factor of three can occur on a time scale of a year - thus the various sections of the survey are complete only for the relevant epoch. Many of their optical counterparts are also variables - variations of up to a factor of 100 can occur on a time scale of one year. It is hoped to make some assessment of the effect of these two factors in the next two years, when second-epoch Parkes surveys will begin and SRC Schmidt plates will overlap the Palomar Sky Survey.

About half the sources in the sample can be identified and of these half are galaxies and half quasars or possible quasars. The identifications with galaxies are based solely on positional coincidence. About $70 \%$ of the identifications with quasars are supported by photometry, two-colour photography or spectroscopy; the remainder are based largely on positional coincidence, although for some there is additional support such as radio or optical variability. The small fraction of the latter objects which may be mis-identified is statistically unimportant. Figure 1 shows histograms of the number of sources 

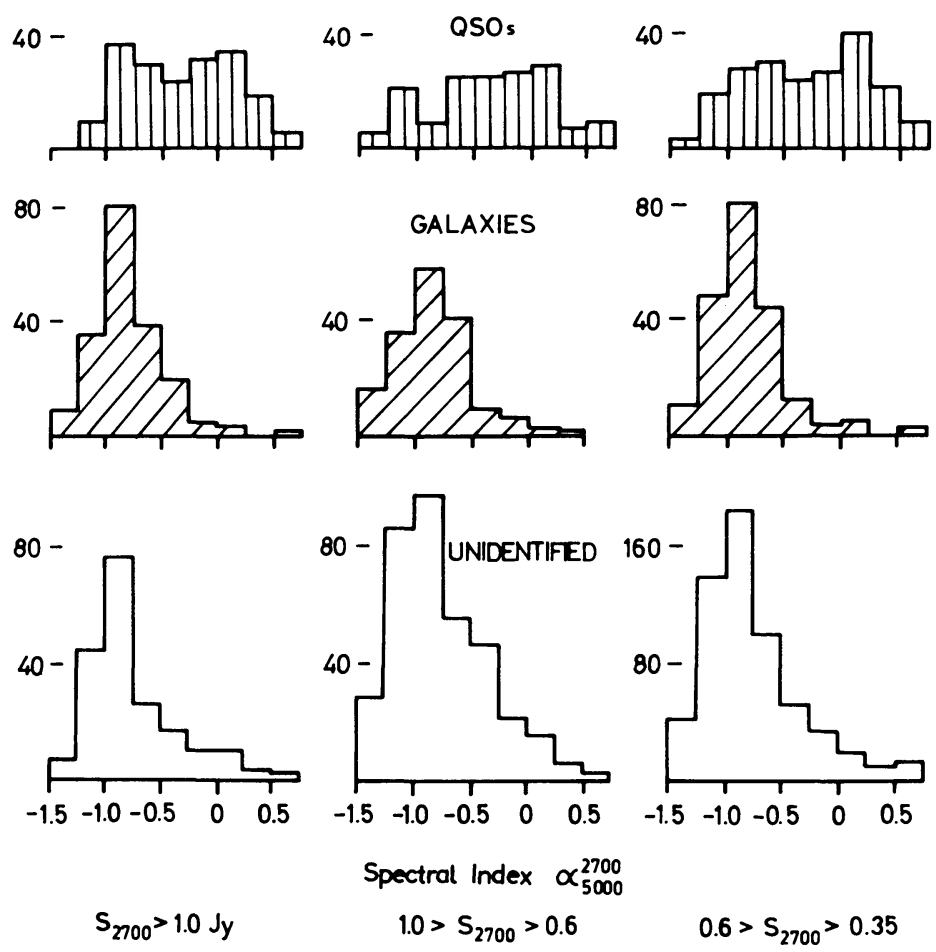

Fig. 1. Distribution of spectral indices between 2700 and $5000 \mathrm{MHz}$ for sources in three identification classes and three ranges of flux density.

in nine spectral index bins for three flux-density ranges and three classes of identifications - quasars, galaxies and unidentified sources. The vertical scales on all these diagrams are the same except for the weak unidentified sources at the lower right, where it is half that of the rest. Obvious from this figure are two results which I first surmised 12 years ago from similar but much less data from the Parkes $408 \mathrm{MHz}$ survey. The spectral index distributions for the QSOs differ markedly from those of the radio galaxies or the unidentified sources. The close parallel between the two latter groups suggests that the majority of unidentified sources are galaxies beyond plate limit. The distributions show little change in form with flux density; however, the fraction of unidentified sources rises rapidly with decreasing flux density. This is perhaps clearer in Figure 2, where the percentage of sources in each identification class is shown in spectral-index bins for the three ranges of flux density. The percentage of identifications - which are largely quasars - is always greater for sources with spectral indices $\alpha>-0.25$. In the strong source group the identified quasars outnumber the unidentified sources by four to one. Certainly some of the latter are quasars which were temporarily subluminous at the epoch of the Palomar Sky Survey; second-epoch plates in the $+4^{\circ}$ zone increased the number of quasar identifications by $10 \%$. Some of 

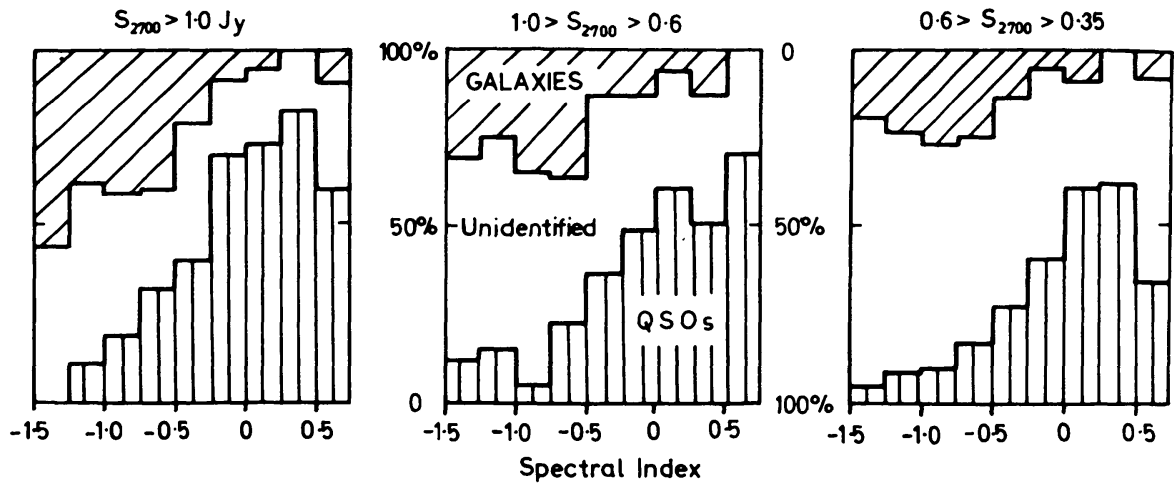

Fig. 2. Percentage of identifications against spectral index for sources in three ranges of flux density at $2700 \mathrm{MHz}$.

the others are undoubtedly objects which were ruled out as quasars because they did not show an ultraviolet excess. Probably less than half the 'missing' identifications arise from this cause and amongst these are likely to be a high fraction of quasars with redshifts greater than 2.5. However, colour is by no means an exclusive indicator for the redshift range of a quasar. In a rather limited sample of $\sim 80$ emission-1ine quasars observed with the Ang1o-Australian 4-m telescope in the past year two out of four with $z>2.3$ have an ultraviolet excess. This sample is however heavily biased towards ultraviolet excess objects. Out of seven neutral stellar objects examined, four have emission-line spectra with $z \ll 2$ and one (discussed 1ater) has an absorption-line spectrum with $z>2.3$. The relationship between colour and redshift is probably influenced as much by the form of the continuum and the presence of absorption features as by the location of strong emission lines.

If the result for the quasars with $\alpha>-0.5$ can be taken as characteristic of quasars with all values of spectral index, then for the strong source group the number of unidentified sources which are below plate limit or are neutral stellar objects is unlikely to be greater than, say, $20 \%$ of the identified quasars. Though this percentage may increase with decreasing flux density it represents an insignificant fraction of all unidentified sources, which, as the similarity in overall spectral index distribution implies, must be galaxies beyond plate limit.

\section{Luminosity Distributions}

The luminosity distributions for the identified radio galaxies, divided into three ranges in flux density and three ranges in spectral index are shown in Figure 3 . It is expected that the numbers in the last magnitude range would be underestimated; near plate limit the ability to distinguish the image of a faint galaxy from that of a faint star is affected both by seeing conditions for the original plate 

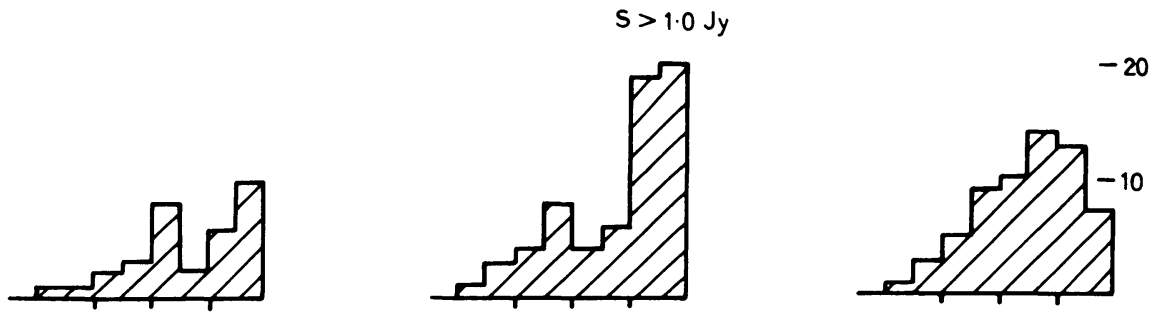

$-20$
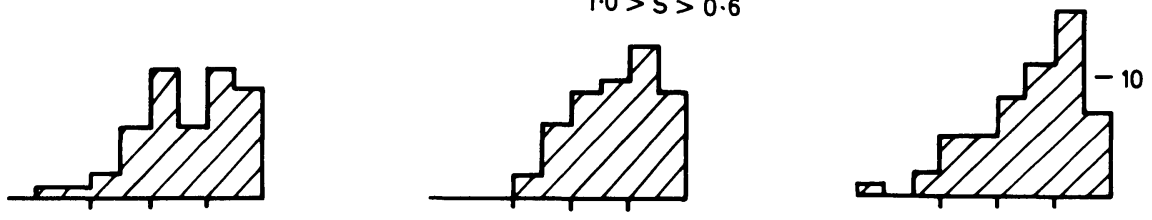

$-20$
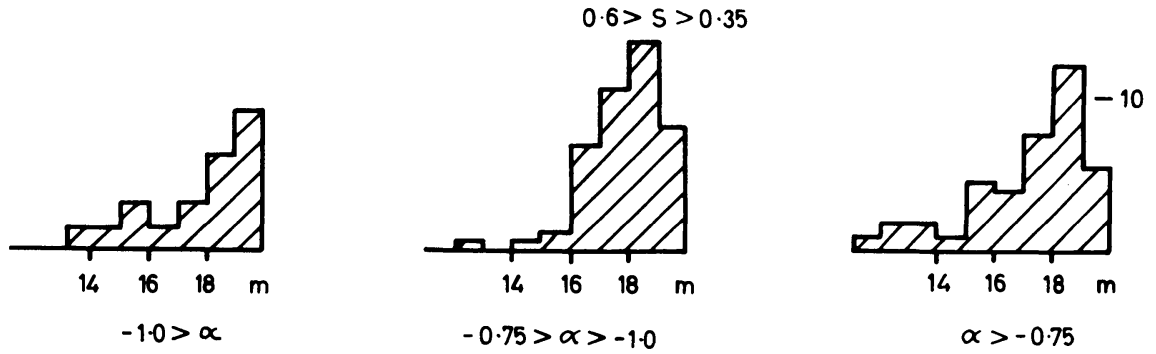

Fig. 3. Luminosity distributions for radio galaxies in three ranges of flux density and three ranges of spectral index.

and the quality of the copy plates or prints. Within the limits of statistical variations there are no significant differences in the luminosity distributions. They are consistent with a radio luminosity function which is extremely broad and a cut-off imposed by the limit of existing sky survey plates. The possible forms of the luminosity distribution beyond plate limit for the three flux density ranges are indicated in Figure 4. These diagrams were constructed using three constraints: (a) that the rise to within 1 magnitude of plate limit continues past the plate limit; (b) that the highest luminosity objects are comparable with the highest already known; and (c) that the number of sources to be accommodated is $80 \%$ of the unidentified sources in each range.

Currently the fractions of identified sources in the three flux density ranges are $0.53,0.39$ and 0.25 . If the extrapolation of the luminosity distributions are realistic a gain of 1.5 magnitudes in plate limit, as may be expected from the SRC Schmidt IIIa-J survey plates, would raise these fractions to $0.83,0.64$ and 0.47 ; identification of such objects would require radio positions accurate to $1^{\prime \prime}$ or 2 " arc. This forecast could be modified by the occurrence of a 

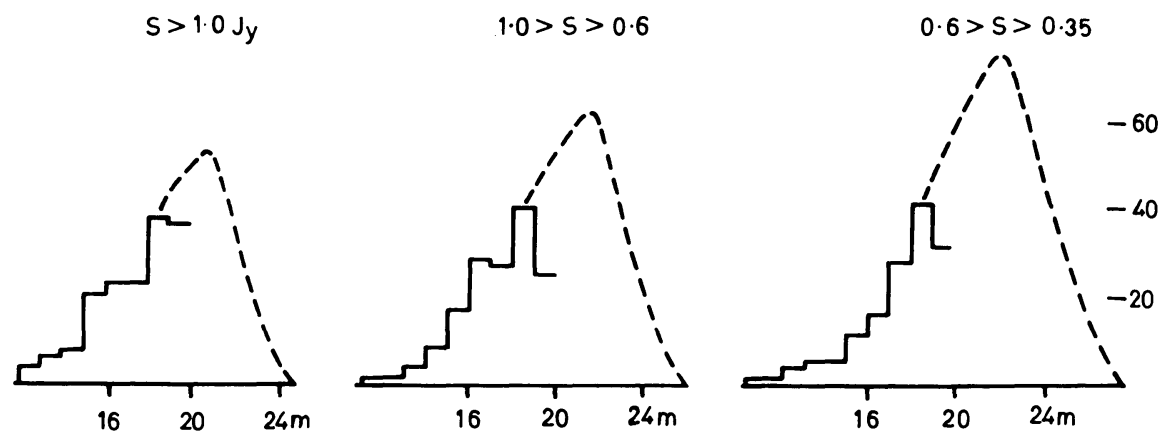

Fig. 4. Luminosity distributions for radio galaxies in three ranges of flux density with an indication of their extrapolation beyond existing sky survey plate limit.

population of faint sources which perhaps have to be associated with a cluster of galaxies rather than the brightest or any individual member. A preliminary examination of a limited number of SRC Schmidt plates of radio positions accurate to only 10" arc suggest that such an effect might be present.

The luminosity distributions for the quasars, again divided into three ranges of flux density and three ranges of spectral index, are shown in Figure 5. Here, although there is a trend for the peaks in the distributions to move towards fainter magnitudes with decreasing flux density, the peaks occur well before plate limit. The addition of a relatively small number of identifications missed because of a colour bias could not radically change the form of these distributions. The occurrence of a peak in the luminosity distribution of the radio quasars has been a result of almost all large-scale identification programs. In strong contrast, the luminosity distributions of quasars selected optically on the basis of ultraviolet excess and in some cases also infrared excess (for example, by Braccesi and his co-workers) appear to rise steeply beyond $18 \mathrm{th}$ magnitude. Unfortunately spectroscopic confirmation is not available for many of the faint optically selected quasars and a large fraction might be galactic stars; it is important that more be investigated spectroscopically. On the assumption that they are correctly identified some explanation is needed of the differerce in the luminosity distribution of the radio quasars and the optically selected quasars. Qualitatively the difference could be understood if the radio emission was fairly closely related to the optical emission for one group of quasars and completely absent in another. There is good evidence for the latter but the situation on the first requirement is not so clear. For the variable quasars, changes in optical brightness appear to have related changes in radio emission and the ratio between radio and optical emission of the radio quasars observationally has a much smaller range than that for the radio galaxies; however, this may be an effect of the inherently smaller ranges in the individual observed quantities. 

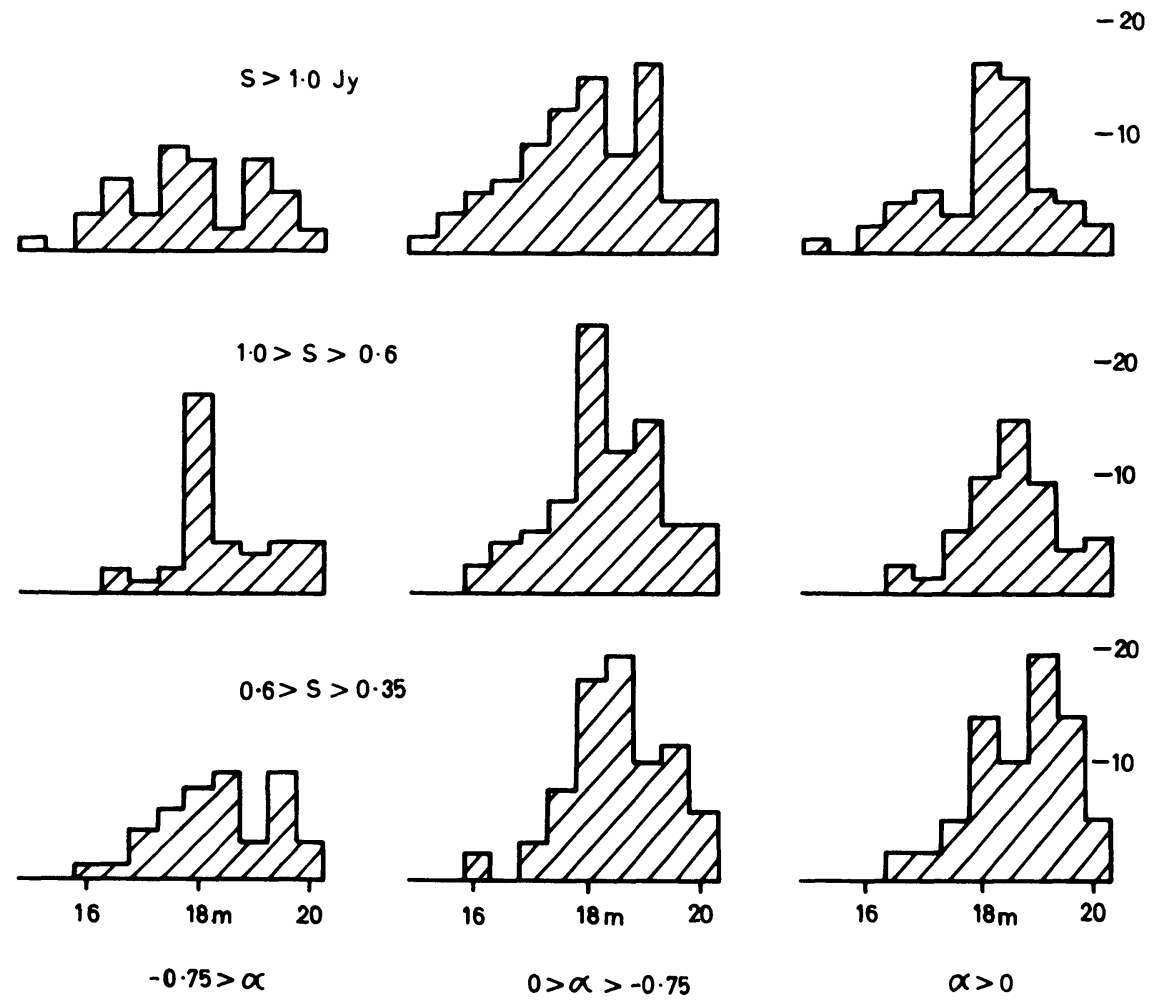

Fig. 5. Luminosity distributions for quasars in three ranges of flux density and three ranges of spectral index.

\section{Radio and Optical Spectra}

From their radio characteristics, the radio quasars can be divided into three classes. The first class contains objects which are in the majority amongst quasars found from low-frequency radio surveys. They have relatively steep power-1aw radio spectra and radio brightness distributions similar in form (and absolute size if redshifts are cosmological) to many bright radio galaxies. These sources occasionally develop weak high-frequency components with time-varying characteristics which agree well with the expanding opaque source models of Kellermann and Pauliny-Toth or Van der Laan. From the rather complete list of redshifts available for the $3 \mathrm{C}$ and $4 \mathrm{C}$ sources it is clear that most of these objects have emission-line spectra.

A second recognizable group of radio quasars have time-averaged spectral indices near zero and about half the sources found in the Parkes $2700 \mathrm{MHz}$ surveys are of this type. Variations in the radio range suggest repeated outbursts of the Kellermann/Pauliny-Toth type, and this has been confirmed in some cases by very-long-baseline interferometry. Variations in flux density at $2700 \mathrm{MHz}$ are typically $30 \%$ in a year and 
in visual brightness 1 magnitude. Collectively, at a given epoch $30 \%$ to $50 \%$ of these objects show no emission lines. Individually, the chance of detecting emission lines appears, rather perversely, to be best when the objects are faintest. Whether the rise in continuum simply swamps the line emission or substantially modifies the gaseous envelope of the quasar provides an interesting subject for future quantitative spectroscopy.

The third class of quasars comprise objects with radio spectra apparently characteristic of opaque synchrotron sources - but which show no variations to within an accuracy of $1 \%$ or $2 \%$ even on a time scale of 10 years. The high stability of these objects is very well documented: they have been used as position calibrators at Parkes for many years and their flux densities have been measured relative to Hydra-A several times each year. Optically these objects have very strong emission lines and it seems possible that the apparent synchrotron cut-off may be in fact due to free-free absorption. The cut-off is not steep enough to be due to a compact source at the centre of an isotropic sphere but could be modelled on the basis of a distributed source within an inhomogeneous medium. A rather logical extension of this idea would be to understand the non-radio quasars as having envelopes which are completely opaque to a11 radio wavelengths. It might help to explain the absence of compact central radio sources in galaxies with obvious nuclear activity. One such case is Pictor-A, where recent observations with the Fleurs synthesis telescope at $1410 \mathrm{MHz}$ shows little or no trace of a central component. The optical spectrum of the nucleus of Pictor-A obtained by R.A.E. Fosbury with the Anglo-Australian 4-m telescope and the Wampler image dissector scanner (Fig. 6) has a spectrum in which the emission lines are extremely strong.

\section{Optical Spectra of Quasars}

Spectra of about 120 quasars have so far been obtained with the Wampler image dissector scanner and of these about 80 yield redshifts ranging from 0.2 to 3.3 . A composite average spectrum for quasars formed from this data shows that the strongest permitted lines after Lyman- $\alpha$ are NV (1240), CIV (1549) and H $\alpha$, and that [OIII] (5007) is the strongest forbidden line. The strength of the NV line is somewhat surprising; its proximity to Ly- $\alpha$ would make it very difficult to distinguish on photographic spectra. One of the most interesting objects studied is 0528-250; a neutral stellar object was indicated as the identification by Parkes measurements and confirmed by observations with the NRAO interferometer. Part of its spectrum is shown in Figure 7; there are no recognizable emission lines, but the many prominent absorption lines give a unique redshift of 2.813 . The absorption spectrum is very similar to that of 3C191 and further investigations with higher resolution should prove very interesting. Figure 7 is shown as $\log F_{\nu} v \log \nu$ and was derived from the original data by scaling against a standard star and re-binning. This treatment allows determination of the optical spectral index of the continuum 


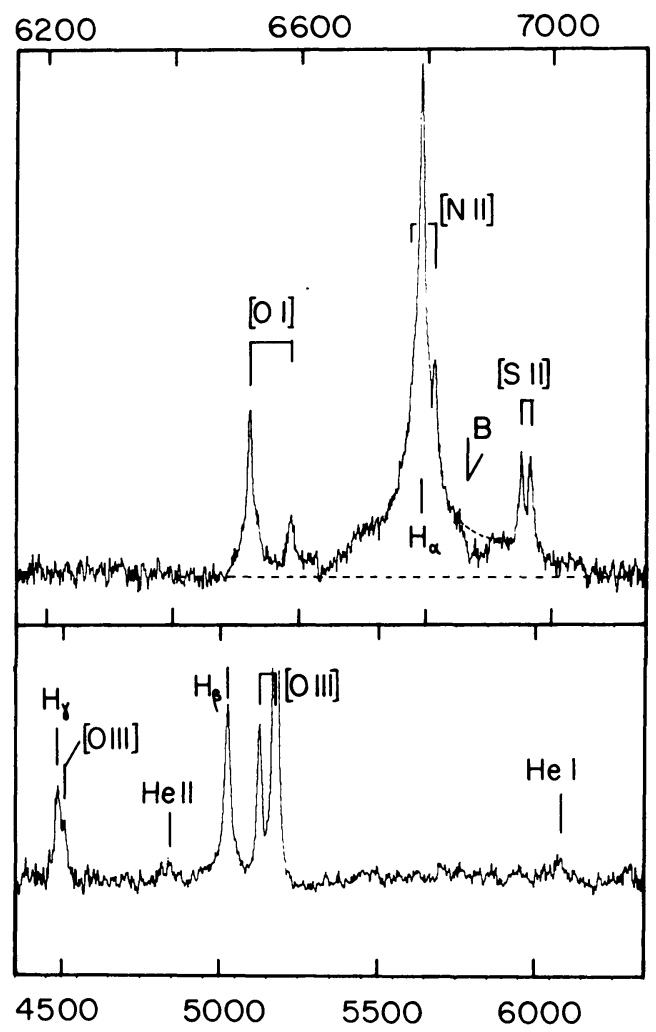

Fig. 6. Spectrum of the radio galaxy Pictor-A obtained by R.A.E. Fosbury with the 3.9-m Anglo-Australian Telescope.

where spectra were obtained at a moderate zenith angle and where they are not obviously curved. A histogram of the spectral indices for 40 such cases is shown in Figure 8. (3C273 was observed to check the validity of the reduction procedure.) The objects shown by the crosshatching, are those with no discernible emission lines. For the objects with redshifts the effects of a continuum $\mathrm{K}$-term (correction for bandwidth compression and shift in wavelength with redshift) shows that on the average the fainter objects are disadvantaged as regards detection to a greater extent than the brighter objects. Similarly the higher redshift objects are disadvantaged relative to the lower redshift objects, with the notable exception of two very high redshift objects with strong UV excess. The two effects appear to be independent.

Non-Cosmological Redshifts

During the past 10 years circumstantial but not compe11ing evidence has been accumulating that the redshifts of quasars and some galaxies have a non-cosmological component. I will very briefly 


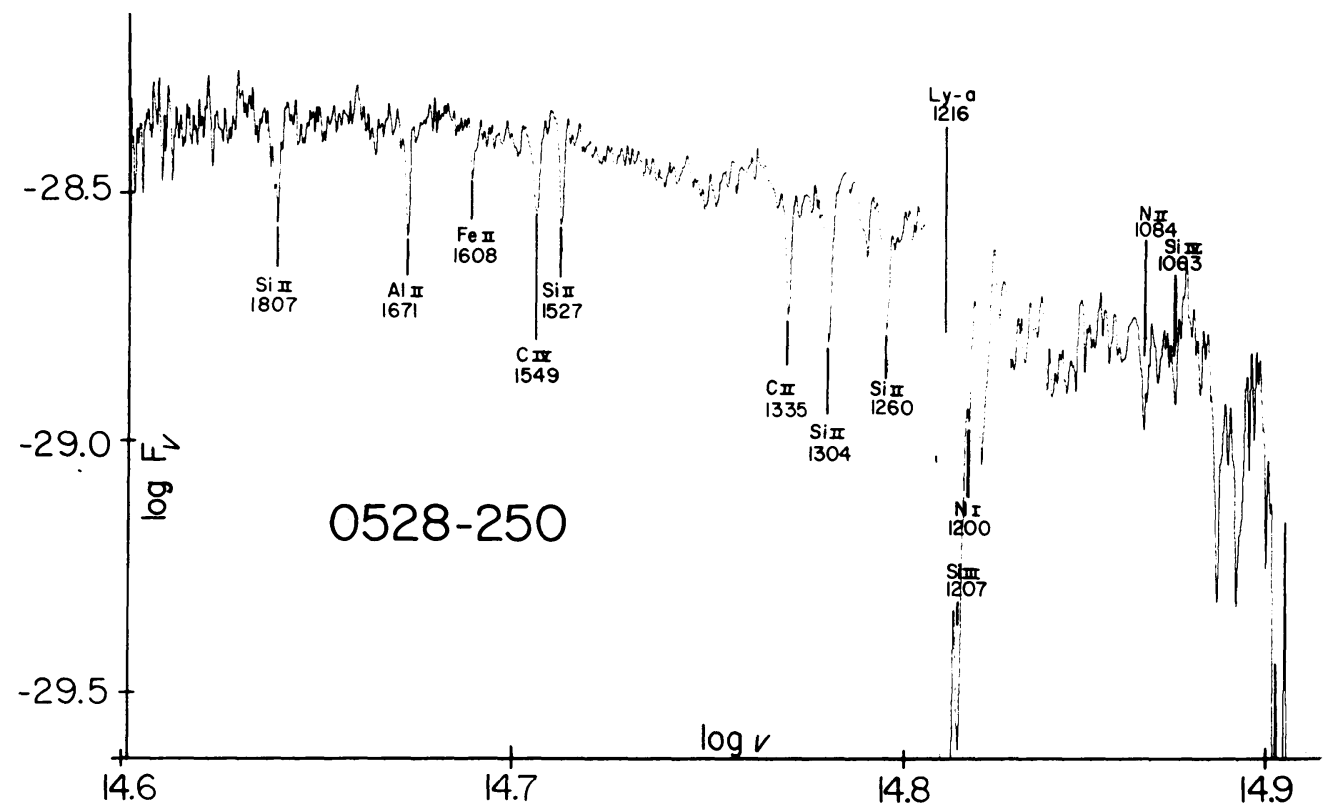

Fig. 7. Part of the spectrum of the quasar 0528-250.

discuss two. One concerns pairing of quasars with bright galaxies. Some years ago Burbidge, $0^{\prime}$ De11 and Strittmatter showed that there was an apparent relationship between the linear separation of a 3C quasar and a nearby galaxy and the redshift of that galaxy. At the same time it was pointed out that similar close associations did not appear in the published identifications of the first zones of the Parkes $2700 \mathrm{MHz}$ catalogue. However, unless radio positions are really precise there is a tendency to associate a radio source with a nearby bright galaxy, since radio and optical centroids do not necessarily coincide. 3C275.1,

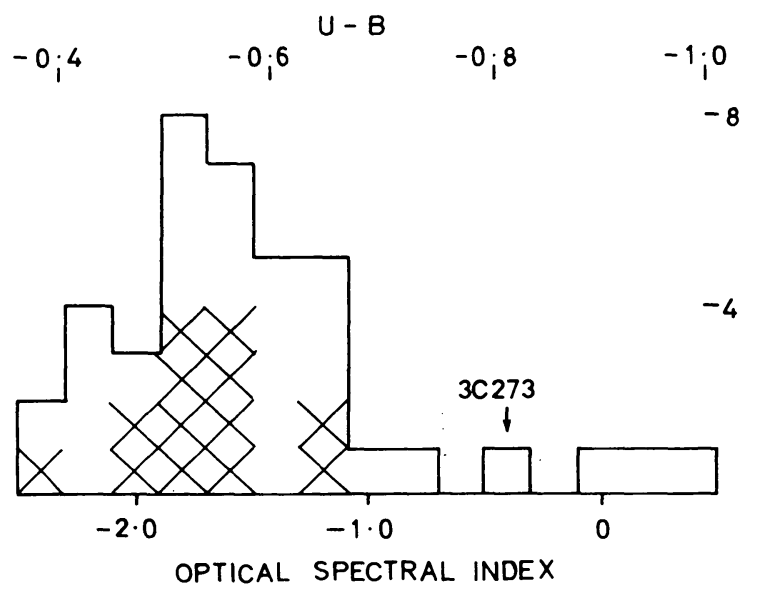

Fig. 8. Distribution of optical spectral indices for 40 quasars. Objects cross-hatched have no emission 1 ines. 
for instance, a quasar in the 3C sample, was originally identified with the nearby galaxy NGC 7413. A number of quasar-bright galaxy pairs are now known in the Parkes sources. For three of these, redshifts have

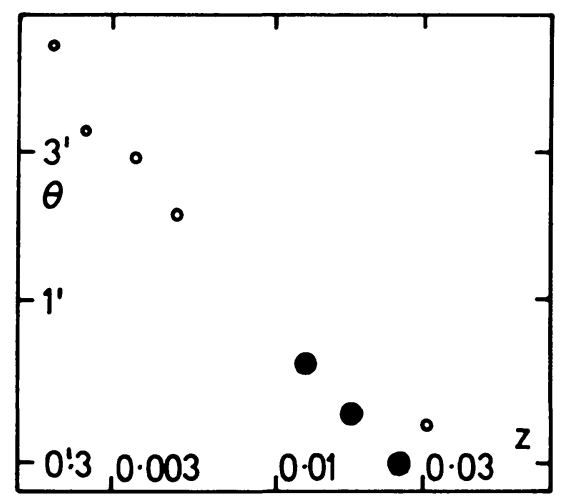

Fig. 9. Galaxy-quasar separation against galaxy redshift (from Burbidge, O'De11 and Strittmatter) with the addition of three new pairs (large circles)。

been measured for the nearby galaxy. They are shown together with the original 3C sample in Figure 9. No comment appears necessary.

In addition to the quasar-galaxy pairs there are a number of quasar-quasar pairs. Most of these were found on two-colour image tube plates taken by B.A. Peterson and me in the positions of radio sources with flat or inverted radio spectra and thus likely to be quasars. Out of a sample of 100 plates, each $2^{\prime}$ arc in radius and containing an object with ultra-violet excess at the position of the radio source, we found eight objects with a second ultra-violet excess object nearby. Spectra by Peterson and myself and by D. and B. Wills have proved that in five out of six cases investigated the non-radio objects are also quasars. Estimates of the surface density of quasars to the limiting magnitude of the plates $(B=19-19.5)$ would predict only one object by chance in the 100 fields. Thus the result appears highly significant. For three of the pairs redshifts are known for both components, and in no cases are the redshifts similar, as is also true of the pair found by Stockton and the pair found by Wampler et a1. If the pairing of quasars with discordant redshifts can be verified in a larger sample, the cosmological origin of the quasar redshifts will be open to question.

I thank my colleagues, B.A. Peterson, A.E. Wright, Ann Savage and D. L. Jauncey for unpublished material discussed in this paper. 


\section{DISCUSSION}

Arp: I have reviewed the quasar-galaxy associations for the Paris Colloquium and find 7 of 8 new cases fall closely along the original Burbidge-Strittmatter-Odell relation with a constant distance of about $20 \mathrm{KPC}$ between the quasar and the galaxy. The new points which Bolton has shown also substantiate this relation and, I think, are mostly in addition to the points I have added.

Wampler: What is the error in your spectral index measurements of the optical spectra of QSO's? In particular are you sure that you have several objects with positive continuum spectral index $(\alpha>0)$ ?

Bolton: Yes, some objects have positive spectral index. Several of these objects have high $z$.

Menon: You mentioned that the ratio of radio to optical flux densities for QSO's is either constant or zero. Does this apply to both the flat and steep spectral index QSO's? In the case of steep spectra QSO's the total flux density need not be correlated with the optical flux density since the radio emission from the central component is smaller than the total flux density. However for the flat spectra QSO's all of the radio emission originates in the central optical object. Hence the ratio of the radio to optical flux density should be computed only when detailed structural information is available, especially for the steep spectra QSO's.

Bolton: I think that it must apply to both types. The relationship might be tighter in the case of the flat spectrum objects in spite of the large variations in both the radio and optical emission.

Webster: Could you please give some idea of any selection effects in your angular separation/redshift diagram?

G. Burbidge: The associations between the QSO's and bright galaxies were investigated statistically and were tested by several methods by Burbidge et al in their paper in 1971 and the results were confirmed using. Monte Carlo methods by Kippenhahn in 1973. It was then found that a plot of $\Delta \theta$ against $z_{G}$ gave the relation just shown by John Bolton and Burbidge, $\mathrm{O}^{\prime} \mathrm{Dell}{ }^{\mathrm{G}}$ \& Strittmatter. We showed in that paper that selection effects would probably work against their observed effect.

Murdoch: You said only one new QSO was discovered on objective prism plates. Does this mean most QSO's discovered by objective prism are radio sources.

Bolton: No, the comparison was not with radio gso but with objects selected as having uv excess on two colour plates. Only one emission line QSO was found which did not correspond to an object already picked out by its uv excess in a sample of 25 , and this had no counterpart on 
the two colour plates - i.e. it had changed in brightness.

van der Laan: The purely positional identification with galaxies, given position of accuracies of $\pm 10 "$, would seem hazardous beyond $\mathrm{m} \sim$ 18. Do you not get a lot of spurious identifications near the plate limit?

Bolton: I don't believe so. The 10" is a limit on the radio position rather than an rms error.

de Felice: Is there any significant difference in the QSO distribution between the Northern and the Southern Hemisphere?

Bolton: The subject was not considered but there is little evidence of significant difference. Any difference is probably due to unexpected obscuration.

\section{PAIRS OF QUASARS}

\section{B.J. Wills and D. Wills}

The probability that the QSO pairs discussed by John Bolton could arise from chance associations of radio QSO's and background radio-quiet QSO's is 1 in 1000, if one uses the background QSO counts given by Setti and Woltjer (Annals N.Y. Acad. Sci., 224, 8 (1973)) from the search by Braccesi et al. (Astr. and Astrophys., 5, 264 (1970)).

With Alan Vomoto, we have made an independent search for QSO pairs under conditions as closely as possible identical to those applying to Bolton and Peterson's search. Two colour $(U, B)$ plates were obtained with the $2.1 \mathrm{~m}$ McDonald telescope, centred at the positions of flatspectrum QSO's, most of them from the Green Bank $5 \mathrm{GHz}$ (S2 and S3) surveys. In nearly 100 fields, we found three UV-excess objects within 2 ' arc of the radio QSO's, and with $B<19.0$. Our spectroscopy showed two of them to be stars, and the third to be a QSO. The expected chance number of QSO's expected is 0.6 . Searching to $\underline{B}=19.5$, the total is 2 QSO's found, compared with 1.3 expected by chance. In neither case are the redshifts of the radio and non-radio QSO's the same.

Thus, our results are quite consistent with the number of objects expected on the basis of current estimates of the surface density of QSO's. Our result can be made consistent with Bolton and Peterson's result, at the expense of increasing current estimates of the surface density of QSO's by a factor of about four.

G. Burbidge: What is the numerical value of the background density of QSO's that you used?

B. Wills: We used Setti and Wotjer's numbers derived from the Braccesi et al. counts, which gives 2.1 or 4.3 per square degree to $B=19.5$ respectively. 
Sanitt: By what factor could the background density of uv excess quasar be increased before there is a contradiction with your results.

B. Wills: If the background density (to $19^{\mathrm{m}} .5$ ) were increased by a factor of 5, our 2 observed QSO's would have a $3 \%$ probability of occurrence. 